\title{
Single deep ultraviolet light emission from boron nitride nanotube film
}

\author{
Lu Hua Li, ${ }^{1,2, a)}$ Ying Chen, ${ }^{1}$ Meng-Yeh Lin, ${ }^{3}$ Alexey M. Glushenkov, ${ }^{1}$ Bing-Ming Cheng, ${ }^{3}$ \\ and Jun $\mathrm{Yu}^{2}$ \\ ${ }^{1}$ Institute for Technology Research and Innovation, Deakin University, Waurn Ponds VIC 3217, Australia \\ ${ }^{2}$ Department of Electronic Materials Engineering, Research School of Physics and Engineering, \\ The Australian National University, Canberra ACT 0200, Australia \\ ${ }^{3}$ National Synchrotron Radiation Research Center, 101 Hsin Ann Road, Hsinchu Science Park, Hsinchu \\ 30076, Taiwan
}

(Received 27 June 2010; accepted 10 September 2010; published online 4 October 2010)

\begin{abstract}
Light in deep ultraviolet (DUV) region has a wide range of applications and the demand for finding DUV light emitting materials at nanoscale is increasingly urgent as they are vital for building miniaturized optic and optoelectronic devices. We discover that boron nitride nanotubes (BNNTs) with a well-crystallized cylindrical multiwall structure and diameters smaller than $10 \mathrm{~nm}$ can have single DUV emission at $225 \mathrm{~nm}(5.51 \mathrm{eV})$. The measured BNNTs are grown on substrate in the form of a thin film. This study suggests that BNNTs may work as nanosized DUV light sources for various applications. (C) 2010 American Institute of Physics. [doi:10.1063/1.3497261]
\end{abstract}

Light in deep ultraviolet (DUV) region has a wide range of applications, such as photolithography, optical data storage, and medical analysis and treatment, and the demand for DUV light emitting materials is increasingly urgent. Recently, single crystal hexagonal boron nitride (hBN) was discovered to have a band-edge luminescence at $215 \mathrm{~nm},{ }^{1,2}$ which made BN materials promising DUV light emitters. Boron nitride nanotubes (BNNTs), nanosized cylinders rolled up seamlessly from $\mathrm{hBN}$ sheets, have a wide band gap (close to $6 \mathrm{eV}$ ) mostly insensitive to tube diameter and chirality changes. ${ }^{3}$ The second harmonic generation and electro-optical efficiency of BNNTs are much larger than those of $\mathrm{hBN}$, and their photoluminescence (PL) quantum yields surpass those of carbon nanotubes (CNTs). ${ }^{4}$ Therefore, BNNTs are potential nanosized DUV light emitters, useful for building miniaturized optic and optoelectronic devices. However, usually weak DUV light emission along with strong and broad 300-330 nm emission was detected from BNNTs (Refs. 5-8) and the exact band gap and optical properties of BNNTs are still in dispute. Here, we report that single DUV emission at $225 \mathrm{~nm}(5.51 \mathrm{eV})$ was found from a film of BNNTs with diameters smaller than $10 \mathrm{~nm}$ and a cylindrical multiwall structure. This finding suggests that BNNTs may work as nanosized DUV light sources.

The PL measurements were conducted on the high flux vacuum ultraviolet beamline BL03A1 of the National Synchrotron Radiation Research Center, Taiwan. The beamline can provide excitation photons with energies $4-40 \mathrm{eV}$. A boron (B) ink coating method was used to produce large area, high density, and high purity BNNT films for measurement. The detailed synthesis and experimental information can be found in Ref. 9.

The scanning electron microscope (SEM) image in Fig. 1(a) shows a BNNT film grown on a silicon dioxide coated silicon wafer $\left(\mathrm{SiO}_{2} / \mathrm{Si}\right)$ substrate. The sample has high purity BNNTs with diameters of 2-10 $\mathrm{nm}$. Transmission electron microscopy (TEM) analysis revealed that these tubes have a well-crystallized cylindrical structure and 2-10 layers of

\footnotetext{
${ }^{\text {a) }}$ Author to whom correspondence should be addressed. Electronic mail: luhua.li@deakin.edu.au.
}

walls [see the inset in Fig. 1(a)]. The luminescence properties of such small BNNTs have not been studied before. Figure 1(b) shows the PL spectra from the film at $10 \mathrm{~K}$ and room temperature after the film was annealed at $150{ }^{\circ} \mathrm{C}$ for $16 \mathrm{~h}$ in vacuum $\left(\sim 10^{-3}\right.$ mbar $)$ to remove any adsorbed gas on nanotube surface. Both spectra exhibit strong single DUV light emission centered at $225 \mathrm{~nm}$. This position does not change from $10 \mathrm{~K}$ to $293 \mathrm{~K}$, indicating that BNNTs have very similar band gap value as $\mathrm{hBN}$, consistent with many recent theoretical and experimental findings. ${ }^{8,10-13}$ However, the $215 \mathrm{~nm}$ peak position has not been observed on these BNNTs. The full width at half maximum (FWHM) of the DUV bands are $30.6 \mathrm{~nm}(0.77 \mathrm{eV})$ and $31.8 \mathrm{~nm}(0.81 \mathrm{eV})$ at $10 \mathrm{~K}$ and $293 \mathrm{~K}$, respectively. The unusually large FWHMs are mainly due to the strong excitonic effects in BNNTs. ${ }^{13,14}$ The asymmetric shapes of the DUV bands suggest the existence of several subbands.

In addition to the strong DUV emission around $225 \mathrm{~nm}$, there are also two weak bands at around $265 \mathrm{~nm}(4.78 \mathrm{eV})$ and $320 \mathrm{~nm}(3.78 \mathrm{eV})$. The first weak band is probably due to bound excitons caused by dislocations and stacking faults. Evidence for this comes from studies of a deformed single crystal of $\mathrm{hBN}:^{15,16}$ after deformation the $215 \mathrm{~nm}$ peak shifted to $227 \mathrm{~nm}$ and a background-like slope appeared in the 250 to $310 \mathrm{~nm}$ range. The weak band at $320 \mathrm{~nm}$ can be attributed to impurities. ${ }^{12,17}$ The luminescence signals shown in Fig. 1(b) are dominantly from BNNTs rather than the $\mathrm{SiO}_{2} / \mathrm{Si}$ substrate or the $\mathrm{hBN}$ particles which might exist in the nanotube film. ${ }^{9}$

The observed single DUV emission can be attributed to the following reasons. The good crystallinity of the small diameter BNNTs is essential. In the case of hBN, the optical emission highly depends on its crystallinity, ${ }^{18}$ and strong DUV emission has only been found on the high quality hBN crystals. ${ }^{1,2}$ It is also true for BNNTs. For comparison, a film of bamboo structured BNNTs with larger diameters (40-80 $\mathrm{nm}$ ) was produced also by the B ink method and tested for emission. This sample shows strong near $300 \mathrm{~nm}$ but weak DUV emission, ${ }^{9}$ due to high level of defects in bamboo structures. In contrast, the well-crystallized cylindrical structure BNNTs with only a few layers of cylindrical walls tend 

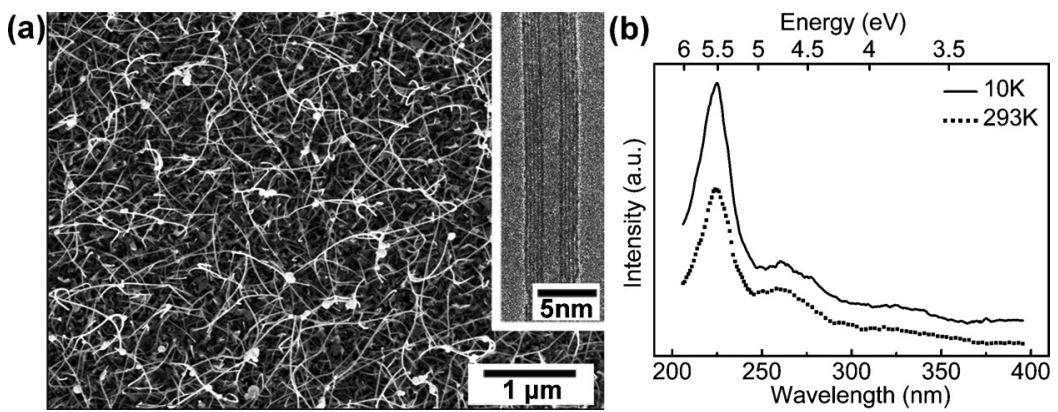

FIG. 1. (a) SEM image of the film of BNNTs with small diameters and TEM image (the inset) showing a well-crystallized multiwall structure; (b) PL spectra from the BNNT film at 10 and $293 \mathrm{~K}$ after vacuum annealing.

to have dramatically lower density of defects so that the strong DUV emission was observed. The high purity of the BNNT film is also vital, because the presence of hBN particle byproducts can give rise to strong near $300 \mathrm{~nm}$ band in luminescence. ${ }^{9}$ In addition, it is also important to remove the atmospheric contamination on BNNT surface, which is discussed below.

Atmospheric gases physisorbed on nanotube surface can have a strong impact on the light emission from the small diameter BNNTs. Figure 2 shows the PL spectrum recorded at $10 \mathrm{~K}$ from the cylindrical BNNT film without the vacuum annealing at $150{ }^{\circ} \mathrm{C}$ [the same sample shown in Fig. 1(a)]. The spectrum consists of a broad band at $315 \mathrm{~nm}$ and a relatively strong DUV emission at $229 \mathrm{~nm}(5.41 \mathrm{eV})$ with FWHM of $28.3 \mathrm{~nm}(0.44 \mathrm{eV})$. A comparison of the $10 \mathrm{~K}$ spectra of the film before (Fig. 2) and after vacuum annealing [Fig. 1(b)] indicates that the absolute intensity of the DUV band increases about $17 \%$ and the broad band around $300 \mathrm{~nm}$ reduces significantly after the cleaning treatment. The DUV band was also blue shifted from 229 to $225 \mathrm{~nm}$ after the cleaning. Both the shift of the DUV band and its broadening may be due to intensity changes of the excitonic subbands in the DUV region. These observed changes must be due to the desorption of the physically attached atmospheric gases on the surface of nanotubes during the vacuum annealing treatment, because chemisorptions, such as chemisorbed oxygen, should not be affected at such low temperature. The vacuum annealing can also slightly improve the emission of large diameter bamboo structure BNNTs, but the effect is much less obvious. ${ }^{9}$ This may indicate that small diameter BNNTs are more prone and sensitive to atmospheric contamination.

It has been found before that the atmospheric gases (oxygen or moisture) can be physisorbed on CNTs and nanosized III-nitride crystals ${ }^{19-21}$ and affect their electronic and luminescence properties. ${ }^{19,22,23}$ Although the physisorption

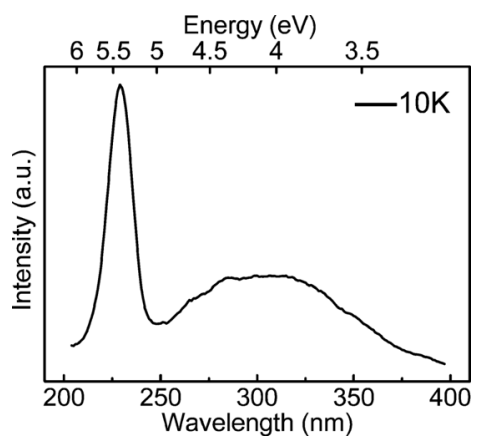

FIG. 2. The $10 \mathrm{~K}$ PL spectrum from the small diameter cylindrical BNNT film before vacuum annealing. of atmospheric gases on BNNTs has never been experimentally tested, one theoretical calculation has predicted that oxygen molecules can physically bind on the surface of even pristine BNNTs. ${ }^{24}$ However, high vacuum alone cannot remove the adsorbed gas. Because although the film was initially placed in a high vacuum chamber at $10^{-7}-10^{-9}$ mbar for measurement, the adsorbed gas was not removed effectively and still affected the luminescence of the small diameter BNNTs (Fig. 2). Only vacuuming at an elevated temperature can clean the surface contamination and consequently improve the DUV emission from BNNTs [Fig. 1(b)], which is similar to the case of CNTs. ${ }^{19}$

The cleaning effect of the vacuum annealing was checked using x-ray energy dispersive spectroscopy (EDS) to semiquantitatively examine the change in oxygen content of the BNNT film before and after the vacuum annealing. The spectra in Fig. 3(a) show a significant decrease in oxygen signal strength after the treatment with an estimated $\sim 60 \%$ reduction in $\mathrm{O}$ content. Thermal gravimetric analyzer (TGA) was also used to monitor the degassing process of BNNTs during heating to $500{ }^{\circ} \mathrm{C}$ in flowing argon $\left(50 \mathrm{ml} \mathrm{min}^{-1}\right)$. The observed TGA curve [Fig. 3(b)] shows a weight drop of about $1.5 \%$ below $250{ }^{\circ} \mathrm{C}$, indicating substantial desorption of oxygen and moisture from the nanotube surfaces. The desorption should be more effective if the annealing treatment is conducted in vacuum for an extended period.

In summary, we report that BNNTs with a wellcrystallized structure and diameters smaller than $10 \mathrm{~nm}$ can have single DUV light emission at $225 \mathrm{~nm}(5.51 \mathrm{eV})$. It was detected from a film of high purity BNNTs. Because of the small sizes of these BNNTs, their luminescence can be easily affected by atmospheric contamination on surface. This study suggests that BNNTs have potential to work as nanosized DUV light emitters for a wide range of applications.
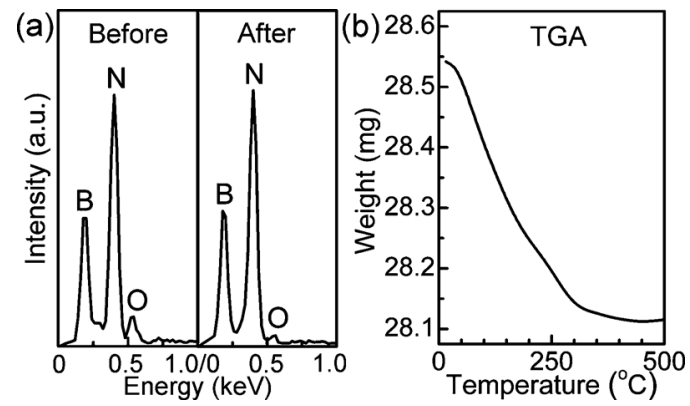

FIG. 3. (a) EDS spectra of the small diameter cylindrical BNNT film before and after the vacuum annealing; (b) TGA curve showing the weight loss of BNNTs during the heating in argon. 
L. H. Li thanks Dr. Peter Lamb of Deakin University, Mr. Renqi Jun and Mr. Lu Jian of Fudan University for various help and Prof. Robert Elliman and Dr. Hua Chen of Australian National University for discussion.

${ }^{1}$ K. Watanabe, T. Taniguchi, and H. Kanda, Nature Mater. 3, 404 (2004). ${ }^{2}$ Y. Kubota, K. Watanabe, O. Tsuda, and T. Taniguchi, Science 317, 932 (2007).

${ }^{3}$ X. Blase, A. Rubio, S. G. Louie, and M. L. Cohen, Europhys. Lett. 28, 335 (1994).

${ }^{4}$ D. Golberg, Y. Bando, C. C. Tang, and C. Y. Zhi, Adv. Mater. 19, 2413 (2007).

${ }^{5}$ C. Y. Zhi, Y. Bando, C. C. Tang, D. Golberg, R. G. Xie, and T. Sekigushi, Appl. Phys. Lett. 86, 213110 (2005).

${ }^{6}$ B. Berzina, L. Trinkler, V. Korsak, R. Krutohvostov, D. L. Carroll, K. B. Ucer, and R. T. Williams, Phys. Status Solidi B 243, 3840 (2006).

${ }^{7}$ P. Jaffrennou, F. Donatini, J. Barjon, J. S. Lauret, A. Maguer, B. AttalTretout, F. Ducastelle, and A. Loiseau, Chem. Phys. Lett. 442, 372 (2007). ${ }^{8}$ H. Chen, Y. Chen, Y. Liu, C. N. Xu, and J. S. Williams, Opt. Mater. (Amsterdam, Neth.) 29, 1295 (2007).

${ }^{9}$ See supplementary material at E-APPLAB-97-091040 for synthesis of BNNT films, experimental details and PL spectra of the substrate, BN particles and a film of bamboo structured BNNTs.

${ }^{10}$ R. Arenal, O. Stephan, M. Kociak, D. Taverna, A. Loiseau, and C. Colliex, Phys. Rev. Lett. 95, 127601 (2005).
${ }^{11}$ L. Wirtz, A. Marini, and A. Rubio, Phys. Rev. Lett. 96, 126104 (2006).

${ }^{12}$ P. Jaffrennou, J. Barjon, T. Schmid, L. Museur, A. Kanaev, J. S. Lauret, C. Y. Zhi, C. Tang, Y. Bando, D. Golberg, B. Attal-Tretout, F. Ducastelle, and A. Loiseau, Phys. Rev. B 77, 235422 (2008).

${ }^{13}$ J. Yu, D. H. Yu, Y. Chen, H. Chen, M. Y. Lin, B. M. Cheng, J. Li, and W. H. Duan, Chem. Phys. Lett. 476, 240 (2009).

${ }^{14}$ P. Jaffrennou, J. Barjon, J. S. Lauret, B. Attal-Tretout, F. Ducastelle, and A. Loiseau, J. Appl. Phys. 102, 116102 (2007).

${ }^{15}$ K. Watanabe, T. Taniguchi, T. Kuroda, and H. Kanda, Appl. Phys. Lett. 89, 141902 (2006).

${ }^{16} \mathrm{~K}$. Watanabe, T. Taniguchi, T. Kuroda, and H. Kanda, Diamond Relat. Mater. 15, 1891 (2006)

${ }^{17}$ K. Watanabe, T. Taniguchi, and H. Kanda, Phys. Status Solidi A 201, 2561 (2004)

${ }^{18}$ C. A. Taylor, S. W. Brown, V. Subramaniam, S. Kidner, S. C. Rand, and R. Clarke, Appl. Phys. Lett. 65, 1251 (1994).

${ }^{19}$ P. G. Collins, K. Bradley, M. Ishigami, and A. Zettl, Science 287, 1801 (2000).

${ }^{20}$ A. Hartschuh, H. N. Pedrosa, L. Novotny, and T. D. Krauss, Science 301, 1354 (2003).

${ }^{21}$ T. Hashizume, R. Nakasaki, S. Ootomo, S. Oyama, and H. Hasegawa, Mater. Sci. Eng., B 80, 309 (2001).

${ }^{22}$ J. Lefebvre, Y. Homma, and P. Finnie, Phys. Rev. Lett. 90, 7401 (2003).

${ }^{23}$ J. Lefebvre, P. Finnie, and Y. Homma, Phys. Rev. B 70, 5419 (2004).

${ }^{24}$ J. Zhang, K. P. Loh, J. W. Zheng, M. B. Sullivan, and P. Wu, Phys. Rev. B 75, 245301 (2007). 